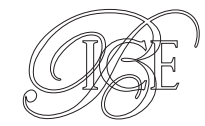

\title{
5. Turismo
}


5.1. NÚMERO DE VIAJEROS, PERNOCTACIONES Y ESTANCIA MEDIA SEGÚN LA CATEGORÍA DEL ESTABLECIMIENTO EN 2017

\begin{tabular}{|c|c|c|c|c|c|c|c|}
\hline \multirow[b]{2}{*}{ Categoría } & \multicolumn{3}{|c|}{ Número de viajeros } & \multicolumn{3}{|c|}{ Número de pernoctaciones } & \multirow{2}{*}{$\begin{array}{l}\text { Estancia } \\
\text { media }\end{array}$} \\
\hline & Total & $\begin{array}{l}\text { Residentes } \\
\text { en España }\end{array}$ & $\begin{array}{l}\text { Residentes en } \\
\text { el extranjero }\end{array}$ & Total & $\begin{array}{l}\text { Residentes } \\
\text { en España }\end{array}$ & $\begin{array}{l}\text { Residentes en } \\
\text { el extranjero }\end{array}$ & \\
\hline TOTAL................................... & 103.496 .998 & 50.227 .660 & 53.269 .338 & 340.037 .164 & 115.036 .697 & 225.000 .467 & 3,29 \\
\hline $\begin{array}{l}\text { Tasa interanual......................... } \\
\text { HOTELES: Estrellas oro }\end{array}$ & 3,66 & 1,38 & 5,91 & 2,68 & 0,70 & 3,72 & $-0,95$ \\
\hline 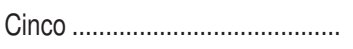 & 5.818 .993 & 1.747 .019 & 4.071 .974 & 20.804 .472 & 3.895 .055 & 16.909 .417 & 3,58 \\
\hline 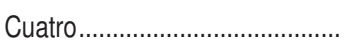 & 48.157 .355 & 21.619 .495 & 26.537 .860 & 170.742 .096 & 50.990 .837 & 119.751 .259 & 3,55 \\
\hline 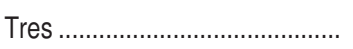 & 28.138 .615 & 13.583 .241 & 14.555 .373 & 99.810 .494 & 33.395 .701 & 66.414 .793 & 3,55 \\
\hline Dos & 8.040 .151 & 5.009 .120 & 3.031 .030 & 19.672 .284 & 10.144 .874 & 9.527 .410 & 2,45 \\
\hline HOSTALES: Estrellas plata & 3.297 .331 & 2.078 .892 & 1.218 .439 & 7.089 .950 & 4.003 .354 & 3.086 .596 & 2,15 \\
\hline 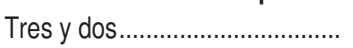 & 5.482 .113 & 3.574 .912 & 1.907 .201 & 11.384 .007 & 6.904 .142 & 4.479 .864 & 2,08 \\
\hline 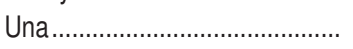 & 4.562 .441 & 2.614 .981 & 1.947 .461 & 10.533 .862 & 5.702 .734 & 4.831 .128 & 2,31 \\
\hline
\end{tabular}

5.2 ESTABLECIMIENTOS, PLAZAS, GRADO DE OCUPACIÓN Y PERSONAL, SEGÚN LA CATEGORÍA DEL ESTABLECIMIENTO, EN 2017

\begin{tabular}{|c|c|c|c|c|}
\hline Categoría & $\begin{array}{l}\text { Número de } \\
\text { establecimientos }\end{array}$ & Plazas & $\begin{array}{l}\text { Grado de } \\
\text { ocupación }\end{array}$ & Personal \\
\hline TOTAL & 14.659 & 1.472 .641 & 61,14 & 210.499 \\
\hline Tasa interanual.. & $-0,68$ & 0,60 & 2,18 & 4,93 \\
\hline \multicolumn{5}{|l|}{ HOTELES: Estrellas oro } \\
\hline 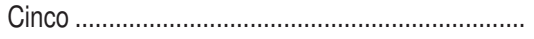 & 284 & 89.675 & 61,97 & 30.271 \\
\hline Cuatro.................... & 2.224 & 667.018 & 67,69 & 103.282 \\
\hline Tres ..................... & 2.473 & 391.803 & 66,85 & 49.805 \\
\hline Dos & 1.854 & 109.337 & 48,20 & 10.112 \\
\hline 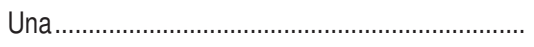 & 1.159 & 48.751 & 39,37 & 4.187 \\
\hline \multicolumn{5}{|l|}{ HOSTALES: Estrellas plata } \\
\hline Tres y dos...... & 2.954 & 85.694 & 36,03 & 6.745 \\
\hline , & 3.711 & 80.363 & 35,66 & 6.096 \\
\hline
\end{tabular}


5.3. NÚMERO DE VIAJEROS, PERNOCTACIONES Y ESTANCIA MEDIA EN 2017

\begin{tabular}{|c|c|c|c|c|c|c|c|}
\hline \multirow[b]{2}{*}{ Meses } & \multicolumn{3}{|c|}{ Número de viajeros } & \multicolumn{3}{|c|}{ Número de pernoctaciones } & \multirow{2}{*}{$\begin{array}{l}\text { Estanci } \\
\text { media }\end{array}$} \\
\hline & Total & $\begin{array}{l}\text { Residentes } \\
\text { en España }\end{array}$ & $\begin{array}{l}\text { Residentes en } \\
\text { el extranjero }\end{array}$ & Total & $\begin{array}{l}\text { Residentes } \\
\text { en España }\end{array}$ & $\begin{array}{l}\text { Residentes en } \\
\text { el extranjero }\end{array}$ & \\
\hline 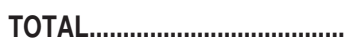 & 103.496 .998 & 50.227 .660 & 53.269 .338 & 340.037 .164 & 115.036 .697 & 225.000 .467 & 3,29 \\
\hline Enero & 4.963 .137 & 2.640 .739 & 2.322 .399 & 14.978 .598 & 5.095 .804 & 9.882 .794 & 3,02 \\
\hline Febrero & 5.659 .692 & 3.141 .988 & 2.517 .705 & 16.046 .230 & 5.965 .374 & 10.080 .856 & 2,84 \\
\hline 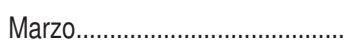 & 6.901 .184 & 3.692 .240 & 3.208 .944 & 20.391 .556 & 7.855 .537 & 12.536 .019 & 2,95 \\
\hline Abril ................. & 9.160 .915 & 4.652 .450 & 4.508 .464 & 27.518 .253 & 10.671 .305 & 16.846 .948 & 3,00 \\
\hline Mayo & 9.840 .760 & 4.171 .327 & 5.669 .434 & 31.546 .445 & 9.051 .738 & 22.494 .707 & 3,21 \\
\hline 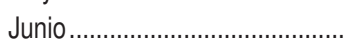 & 10.608 .777 & 4.765 .151 & 5.843 .626 & 36.550 .738 & 10.827 .372 & 25.723 .366 & 3,45 \\
\hline 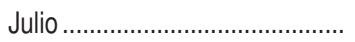 & 11.810 .375 & 5.405 .168 & 6.405 .208 & 43.651 .610 & 14.304 .525 & 29.347 .085 & 3,70 \\
\hline Agosto & 12.301 .182 & 5.932 .359 & 6.368 .823 & 46.720 .017 & 17.031 .650 & 29.688 .367 & 3,80 \\
\hline 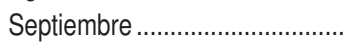 & 10.926 .097 & 4.745 .234 & 6.180 .862 & 38.024 .241 & 11.312 .979 & 26.711 .263 & 3,48 \\
\hline 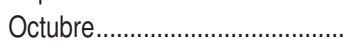 & 9.461 .838 & 4.296 .732 & 5.165 .106 & 30.936 .781 & 9.235 .163 & 21.701 .619 & 3,27 \\
\hline 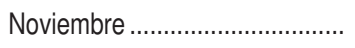 & 6.070 .978 & 3.322 .024 & 2.748 .955 & 17.492 .340 & 6.701 .111 & 10.791 .228 & 2,88 \\
\hline 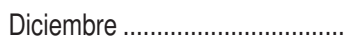 & 5.792 .061 & 3.462 .247 & 2.329 .814 & 16.180 .355 & 6.984 .139 & 9.196 .216 & 2,79 \\
\hline
\end{tabular}

5.4 ESTABLECIMIENTOS, PLAZAS, GRADO DE OCUPACIÓN Y PERSONAL EN 2017

\begin{tabular}{|c|c|c|c|c|}
\hline Meses & $\begin{array}{c}\text { Número } \\
\text { de establecimientos }\end{array}$ & Número de plazas & $\begin{array}{c}\text { Grado de ocupación } \\
(\%)\end{array}$ & Personal empleado \\
\hline TOTAL & 14.659 & 1.472 .641 & 61,14 & 210.499 \\
\hline 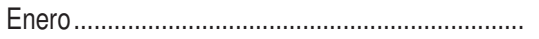 & 12.023 & 1.067 .535 & 44,81 & 146.063 \\
\hline Febrero & 12.659 & 1.148 .568 & 49,29 & 156.259 \\
\hline Marzo....................... & 13.548 & 1.257 .983 & 51,81 & 171.817 \\
\hline Abril ............................. & 14.995 & 1.459 .228 & 61,36 & 201.330 \\
\hline 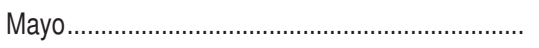 & 15.855 & 1.711 .596 & 58,49 & 237.592 \\
\hline Junio ................... & 16.351 & 1.772 .422 & 66,45 & 256.165 \\
\hline Julio ..................... & 16.774 & 1.804 .339 & 73,14 & 271.651 \\
\hline 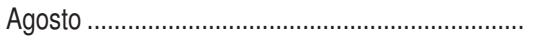 & 16.903 & 1.811 .045 & 77,46 & 275.848 \\
\hline 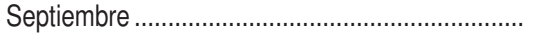 & 16.384 & 1.787 .221 & 69,33 & 263.488 \\
\hline 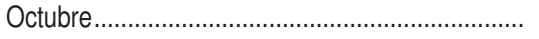 & 15.174 & 1.609 .459 & 60,84 & 228.942 \\
\hline 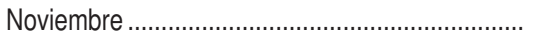 & 12.827 & 1.143 .150 & 50,55 & 161.298 \\
\hline Diciembre & 12.417 & 1.099 .149 & 46,90 & 155.536 \\
\hline
\end{tabular}


5.5. VIAJEROS, PERNOCTACIONES Y ESTANCIA MEDIA EN 2017

(Datos por provincias y CCAA)

\begin{tabular}{|c|c|c|c|c|c|c|c|}
\hline & & mero de viaje & & Nún & o de pernoct & iones & \\
\hline & Total & $\begin{array}{c}\text { Residentes } \\
\text { en España }\end{array}$ & $\begin{array}{c}\text { Residentes } \\
\text { en el extranjero }\end{array}$ & Total & $\begin{array}{c}\text { Residentes } \\
\text { en España }\end{array}$ & $\begin{array}{c}\text { Residentes } \\
\text { en el extranjero }\end{array}$ & ledia \\
\hline 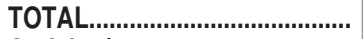 & 103.496 .998 & 50.227 .660 & 53.269 .338 & 340.037 .164 & 115.036 .697 & 225.000 .467 & 3,29 \\
\hline Andalucía ...................................... & 18.334.567 & 9.465 .554 & 8.869 .013 & 52.442 .728 & 23.123.798 & 29.318 .930 & 2,86 \\
\hline Almería ......................................... & 1.410 .347 & 1.052 .682 & 357.665 & 5.549 .307 & 3.617 .595 & 1.931 .711 & 3,93 \\
\hline 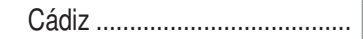 & 2.570 .967 & 1.612 .270 & 958.697 & 7.676 .835 & 4.120 .972 & 3.555 .863 & 2,99 \\
\hline 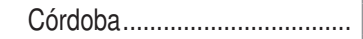 & 1.231 .155 & 710.551 & 520.604 & 1.990 .894 & 1.205 .861 & 785.034 & 1,62 \\
\hline Granada...... & 2.826 .822 & 1.479 .088 & 1.347 .734 & 5.874 .164 & 3.199 .825 & 2.674 .339 & 2,08 \\
\hline Huelva ........ & 1.041 .787 & 767.412 & 274.375 & 4.077 .485 & 2.560 .007 & 1.517.478 & 3,91 \\
\hline Jaén...... & 542.753 & 460.188 & 82.565 & 956.818 & 826.292 & 130.526 & 1,76 \\
\hline Málaga.......................................... & 5.345 .736 & 1.902 .214 & 3.443 .521 & 19.883 .533 & 4.965 .642 & 14.917 .891 & 3,72 \\
\hline Sevilla ....................................... & 3.365 .000 & 1.481 .148 & 1.883 .851 & 6.433 .691 & 2.627 .603 & 3.806 .088 & 1,91 \\
\hline 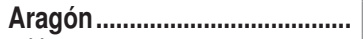 & 2.879 .292 & 2.271 .686 & 607.606 & 5.581 .476 & 4.500 .742 & 1.080 .735 & 1,94 \\
\hline Huesca …................................. & 900.271 & 745.021 & 155.250 & 2.114.389 & 1.779 .682 & 334.707 & 2,35 \\
\hline Teruel .... & 441.062 & 391.294 & 49.768 & 806.679 & 703.817 & 102.862 & 1,83 \\
\hline Zaragoza ... & 1.537 .958 & 1.135 .371 & 402.587 & 2.660 .409 & 2.017 .243 & 643.166 & 1,73 \\
\hline Asturias, Principado de ........... & 1.747 .764 & 1.463 .550 & 284.214 & 3.541 .557 & 3.003 .276 & 538.281 & 2,03 \\
\hline Balears, Illes................................ & 10.105 .299 & 1.332 .268 & 8.773 .030 & 59.559 .865 & 5.110 .046 & 54.449 .819 & 5,89 \\
\hline Canarias ....................................... & 9.776 .121 & 1.863 .512 & 7.912 .609 & 70.946 .126 & 7.972 .917 & 62.973 .210 & 7,26 \\
\hline 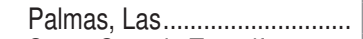 & 5.929 .415 & 970.784 & 4.958 .630 & 44.243 .792 & 4.193.346 & 40.050 .446 & 7,46 \\
\hline Santa Cruz de Tenerife ........... & 3.846 .706 & 892.728 & 2.953 .979 & 26.702 .335 & 3.779 .571 & 22.922 .764 & 6,94 \\
\hline 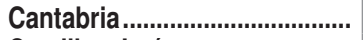 & 1.217.133 & 968.437 & 248.696 & 2.808 .820 & 2.290 .469 & 518.351 & 2,31 \\
\hline Castilla y León ............................. & 4.953 .640 & 3.772 .949 & 1.180 .691 & 8.178 .049 & 6.425 .997 & 1.752 .052 & 1,65 \\
\hline 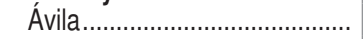 & 391.649 & 333.721 & 57.928 & 609.694 & 521.712 & 87.983 & 1,56 \\
\hline Burgos $\ldots \ldots \ldots \ldots \ldots \ldots \ldots \ldots \ldots \ldots \ldots$ & 903.957 & 581.212 & 322.745 & 1.340 .312 & 928.836 & 411.476 & 1,48 \\
\hline León........ & 786.474 & 624.138 & 162.336 & 1.330 .187 & 1.085 .938 & 244.250 & 1,69 \\
\hline Palencia.... & 190.730 & 137.104 & 53.626 & 337.577 & 260.685 & 76.892 & 1,77 \\
\hline Salamanca................................ & 989.782 & 683.249 & 306.533 & 1.688 .832 & 1.235 .131 & 453.700 & 1,71 \\
\hline 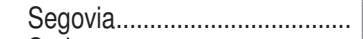 & 467.773 & 375.647 & 92.126 & 813.283 & 643.353 & 169.930 & 1,74 \\
\hline Soria ............. & 242.874 & 223.859 & 19.015 & 434.454 & 401.790 & 32.664 & 1,79 \\
\hline Valladolid ..................................... & 740.519 & 601.482 & 139.036 & 1.240 .791 & 1.004 .929 & 235.862 & 1,68 \\
\hline Zamora $\ldots \ldots \ldots \ldots \ldots \ldots \ldots \ldots \ldots \ldots$ & 239.882 & 212.537 & 27.345 & 382.919 & 343.623 & 39.295 & 1,60 \\
\hline Castilla-La Mancha .................... & 2.184 .340 & 1.781 .202 & 403.138 & 3.670 .705 & 3.029 .070 & 641.635 & 1,68 \\
\hline Albacete...................................... & 342.418 & 312.510 & 29.908 & 681.575 & 616.574 & 65.001 & 1,99 \\
\hline Ciudad Real................................ & 421.486 & 371.964 & 49.522 & 716.191 & 637.101 & 79.089 & 1,70 \\
\hline Cuenca $\ldots \ldots \ldots \ldots \ldots \ldots \ldots \ldots \ldots \ldots$ & 295.896 & 260.793 & 35.103 & 517.456 & 452.046 & 65.410 & 1,75 \\
\hline Guadalajara ................................ & 296.205 & 256.036 & 40.170 & 469.305 & 400.572 & 68.734 & 1,58 \\
\hline Toledo............. & 828.335 & 579.900 & 248.435 & 1.286 .179 & 922.777 & 363.402 & 1,55 \\
\hline Cataluña ..................................... & 19.748 .837 & 7.455 .160 & 12.293 .677 & 57.062 .886 & 16.496 .769 & 40.566 .116 & 2,89 \\
\hline 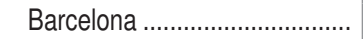 & 12.066 .777 & 3.376 .840 & 8.689 .937 & 32.097 .920 & 6.670 .171 & 25.427 .749 & 2,66 \\
\hline 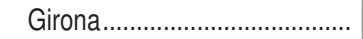 & 3.742 .475 & 1.638 .873 & 2.103 .601 & 12.077 .309 & 3.908 .833 & 8.168 .476 & 3,23 \\
\hline 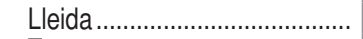 & 862.331 & 713.845 & 148.486 & 1.848 .993 & 1.532 .090 & 316.903 & 2,14 \\
\hline Tarragona .................................... & 3.077 .255 & 1.725 .603 & 1.351 .653 & 11.038 .664 & 4.385 .675 & 6.652 .988 & 3,59 \\
\hline Comunitat Valenciana .............. & 8.698 .045 & 5.148 .388 & 3.549 .657 & 29.154 .416 & 15.376.254 & 13.778 .161 & 3,35 \\
\hline Alicante/Alacant........................... & 4.377 .953 & 2.339 .685 & 2.038 .268 & 18.013 .309 & 8.083 .580 & 9.929 .730 & 4,11 \\
\hline Castellón/Castelló.................... & 1.212 .388 & 1.014 .846 & 197.542 & 3.903.232 & 3.276.971 & 626.262 & 3,22 \\
\hline Valencia/València ..................... & 3.107 .704 & 1.793 .857 & 1.313 .847 & 7.237 .874 & 4.015 .704 & 3.222 .170 & 2,33 \\
\hline Extremadura................................. & 1.376 .876 & 1.143 .796 & 233.079 & 2.376 .959 & 2.041 .900 & 335.059 & 1,73 \\
\hline 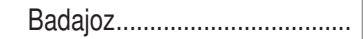 & 685.401 & 554.876 & 130.525 & 1.143 .646 & 968.938 & 174.708 & 1,67 \\
\hline 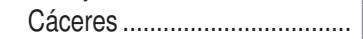 & 691.475 & 588.920 & 102.555 & 1.233 .313 & 1.072 .962 & 160.351 & 1,78 \\
\hline Galicia........................................ & 4.477.689 & 3.177.247 & 1.300 .442 & 8.778 .079 & 6.663 .770 & 2.114.309 & 1,96 \\
\hline Coruña, A ................................. & 2.064 .871 & 1.315 .584 & 749.286 & 3.694 .121 & 2.486 .433 & 1.207 .688 & 1,79 \\
\hline 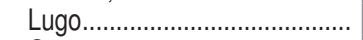 & 667.449 & 465.369 & 202.079 & 1.083 .971 & 848.552 & 235.419 & 1,62 \\
\hline 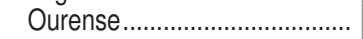 & 307.377 & 273.984 & 33.393 & 609.147 & 535.786 & 73.361 & 1,98 \\
\hline Pontevedra ............................... & 1.437 .992 & 1.122 .309 & 315.683 & 3.390 .840 & 2.793 .000 & 597.840 & 2,36 \\
\hline Madrid, Comunidad de............. & 11.964 .315 & 6.244 .411 & 5.719 .904 & 23.776 .624 & 10.662 .074 & 13.114 .550 & 1,99 \\
\hline Murcia, Región de...................... & 1.293 .969 & 1.015 .540 & 278.429 & 3.133 .007 & 2.361 .274 & 771.733 & 2,42 \\
\hline Navarra, Comunidad Foral de... & 1.017 .697 & 738.925 & 278.772 & 1.900 .637 & 1.440 .280 & 460.357 & 1,87 \\
\hline País Vasco..................................... & 2.992 .713 & 1.816 .803 & 1.175 .909 & 5.745 .247 & 3.435 .866 & 2.309 .381 & 1,92 \\
\hline 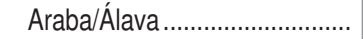 & 421.476 & 296.771 & 124.706 & 773.728 & 548.094 & 225.634 & 1,84 \\
\hline Bizkaia ........................................ & 1.465 .068 & 898.184 & 566.884 & 2.745 .433 & 1.653 .601 & 1.091 .831 & 1,87 \\
\hline 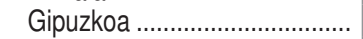 & 1.106 .168 & 621.849 & 484.320 & 2.226 .086 & 1.234 .171 & 991.916 & 2,01 \\
\hline Rioja, La........................................ & 586.936 & 476.020 & 110.916 & 1.039 .974 & 853.525 & 186.449 & 1,77 \\
\hline Ceuta.............................................. & 79.915 & 50.156 & 29.759 & 184.742 & 123.699 & 61.043 & 2,31 \\
\hline Melilla.......................................... & 61.851 & 42.056 & 19.795 & 155.268 & 124.971 & 30.297 & 2,51 \\
\hline
\end{tabular}


5.6. VIAJEROS Y PERNOCTACIONES SEGÚN PAÍS DE RESIDENCIA EN 2017

\begin{tabular}{|c|c|c|c|c|}
\hline \multirow{2}{*}{ País de residencia } & \multicolumn{2}{|c|}{ Número de viajeros } & \multicolumn{2}{|c|}{ Número de pernoctaciones } \\
\hline & Total & $\%$ & Total & $\%$ \\
\hline 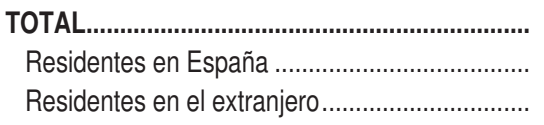 & $\begin{array}{r}103.496 .998 \\
50.227 .660 \\
53.269 .338\end{array}$ & $\begin{array}{r}100,00 \\
48,53 \\
51,47\end{array}$ & $\begin{array}{l}340.037 .164 \\
115.036 .697 \\
225.000 .467\end{array}$ & $\begin{array}{r}100,00 \\
33,83 \\
66,17\end{array}$ \\
\hline TOTAL residentes en el extranjero........................ & 53.269 .338 & 100,00 & 225.000 .467 & 100,00 \\
\hline TOTAL residentes en UE (sin España)............... & 37.713 .945 & 70,80 & 181.951 .826 & 80,87 \\
\hline Alemania... & 8.026 .683 & 15,07 & 50.307 .926 & 22,36 \\
\hline Austria & 462.742 & 0,87 & 2.074 .893 & 0,92 \\
\hline Bélgica & 1.382 .482 & 2,60 & 6.598 .432 & 2,93 \\
\hline Dinamarca & 661.772 & 1,24 & 3.281 .225 & 1,46 \\
\hline 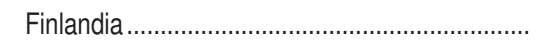 & 399.467 & 0,75 & 1.977 .750 & 0,88 \\
\hline Francia. & 5.646 .271 & 10,60 & 17.204 .065 & 7,65 \\
\hline Grecia & 121.632 & 0,23 & 319.613 & 0,14 \\
\hline Irlanda & 860.966 & 1,62 & 3.953 .461 & 1,76 \\
\hline Italia & 2.934 .136 & 5,51 & 9.318 .958 & 4,14 \\
\hline Luxemburgo & 88.580 & 0,17 & 469.178 & 0,21 \\
\hline 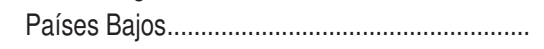 & 2.072 .031 & 3,89 & 9.662 .706 & 4,29 \\
\hline 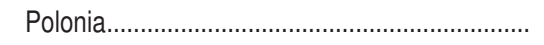 & 878.909 & 1,65 & 4.437 .606 & 1,97 \\
\hline 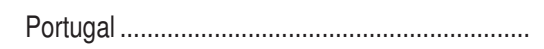 & 1.414 .344 & 2,66 & 3.728 .306 & 1,66 \\
\hline Reino Unido & 10.208 .186 & 19,16 & 57.101 .552 & 25,38 \\
\hline 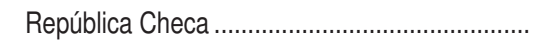 & 257.414 & 0,48 & 1.299 .642 & 0,58 \\
\hline 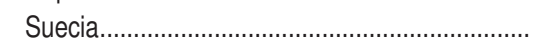 & 1.176 .501 & 2,21 & 6.251 .317 & 2,78 \\
\hline 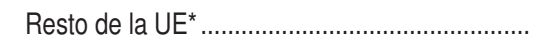 & 1.121 .829 & 2,11 & 3.965 .195 & 1,76 \\
\hline 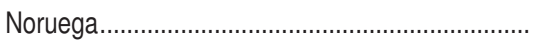 & 625.699 & 1,17 & 3.349 .918 & 1,49 \\
\hline Rusia ........ & 945.236 & 1,77 & 4.846 .813 & 2,15 \\
\hline 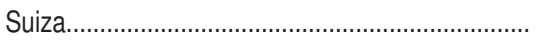 & 1.022 .121 & 1,92 & 4.460 .863 & 1,98 \\
\hline 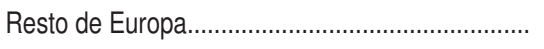 & 1.038 .445 & 1,95 & 3.623 .615 & 1,61 \\
\hline Japón. & 601.829 & 1,13 & 1.111 .270 & 0,49 \\
\hline 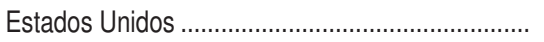 & 2.798 .400 & 5,25 & 6.559 .024 & 2,92 \\
\hline Resto de América & 3.318 .910 & 6,23 & 7.914 .088 & 3,52 \\
\hline Países africanos & 752.750 & 1,41 & 1.901 .063 & 0,84 \\
\hline Resto del mundo & 4.452 .003 & 8,36 & 9.281 .987 & 4,13 \\
\hline
\end{tabular}

